Robotics \& Automation Society

Networking the World

PROCEEDINGS OF THE SECOND INTERNATIONAL WORKSHOP ON ROBOT MOTION AND CONTROL
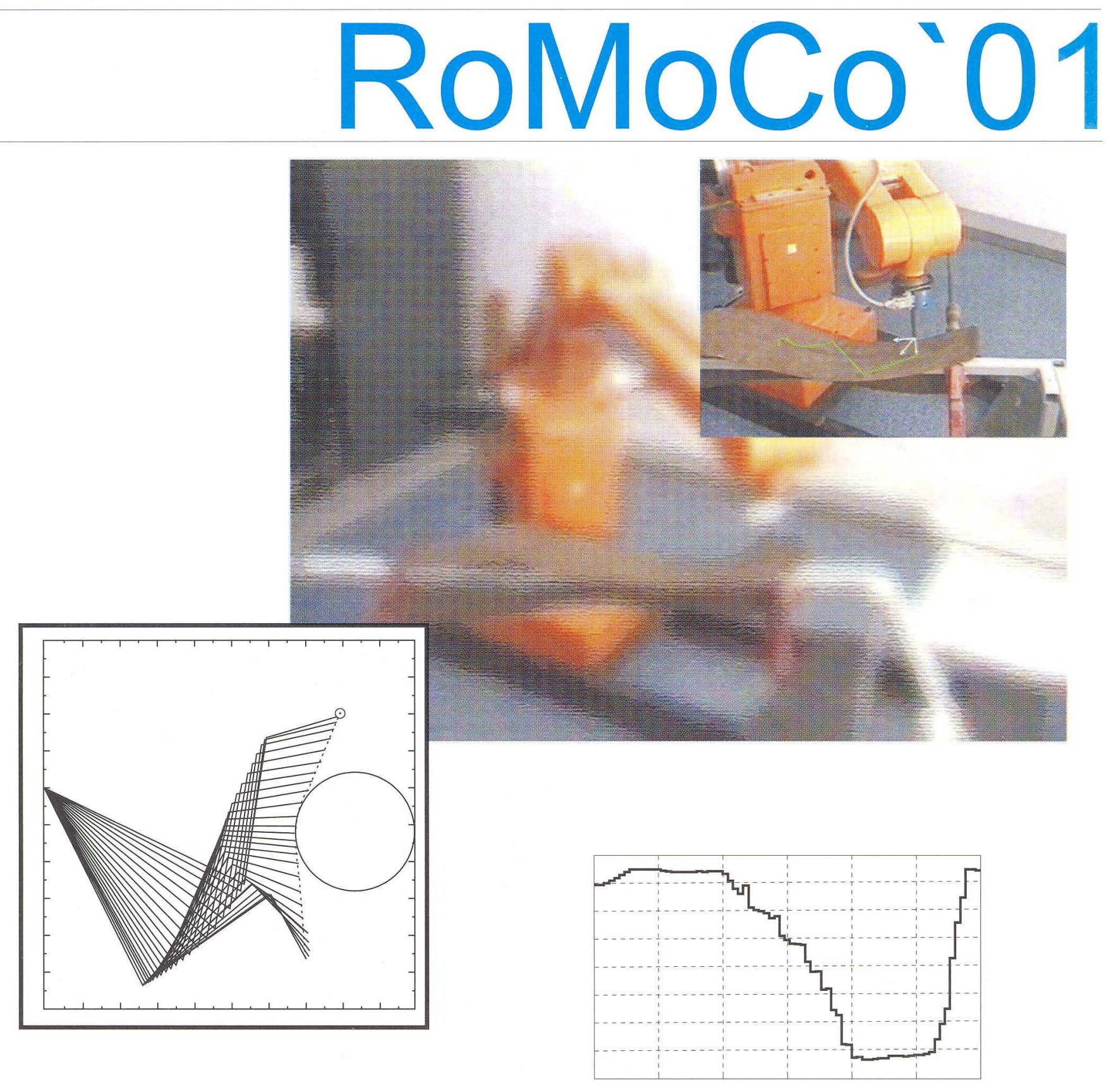

UNIVERSITY OF ZIELONA GORA POZNAŃ UNIVERSITY OF TECHNOLOGY 



\section{Performance Analysis of Multi-Legged Systems}

\author{
Manuel F. Silva, J. A. Tenreiro Machado \\ Dept. of Electrical Engineering \\ Institute of Engineering of Porto \\ Rua Dr. Antonio Bernardino de Almeida, \\ 4200-072 Porto, Portugal \\ Email: $\{\mathrm{mfs}, \mathrm{jtm}\} @$ dee.isep.ipp.pt
}

\author{
António M. Lopes \\ Dept. of Mechanical Engineering \\ Faculty of Engineering of Porto \\ Rua Dr. Roberto Frias, \\ 4200-465 Porto, Portugal \\ Email: aml@fe.up.pt
}

\begin{abstract}
This paper studies periodic gaits of multi-legged robot locomotion systems based on dynamic models. The purpose is to determine the system performance during walking and the best set of locomotion variables that minimizes the optimization indices. For that objective the prescribed motion of the robot is completely characterized in terms of several locomotion variables such as gait, duty factor, body height, step length, stroke pitch, foot clearance, link lengths, body and legs mass and cycle time. In this perspective, we formulate four performance measures of the walking robot namely, the locomobility of the foot, the mean absolute power, the mean power dispersion and the mean power lost in the joint actuators per walking distance. A set of modelbased experiments reveals the influence of the locomotion variables in the proposed indieen.
\end{abstract}

Keywords - legged locomotion, robotics, simulation

\section{INTRODUCTION}

Walking machines allow locomotion in terrain inaccessible to other type of vehicles, since they do not need a continuous support surface [1],[2]. On the other hand, the requirements for leg coordination and control impose difficulties beyond those encountered in wheeled robots [3],[4]. Gait selection is a research area requiring an appreciable modeling effort for improvement of mobility with legs in unstructured environments. Previous studies focused in the structure and selection of locomotion modes [5],[6]. Nevertheless, there are different optimization criteria such as energy efficiency, stability, velocity, comfort, mobility and environmental impact [7],[8]. With these facts in mind, a simulation model for multileg locomotion systems was developed, for several periodic gaits [9]-[12]. This study intends to generalize previous work [13]-[16] through the formulation of several kinematic and dynamic indices, measuring the system locomobility, the average power during different walking trajectories, the standard deviation of the power consumption and the power lost in the joint actuators along the space-time walking cycle.

The foot and body trajectories are analyzed in what concerns its variation with the gait, duty factor, step length, maximum foot clearance and body height. Several simulation experiments reveal the system configuration and the type of the movements that lead to a better mechanical implementation for a given locomotion mode, from the viewpoint of the proposed indices.

Bearing these facts in mind, the paper is organized as follows. Section two introduces the model for a multi-legged robot and the motion planning algorithms. Section three formulates the optimizing indices and section four develops a set of experiments that reveal the influence of the system parameters in the periodic gaits, respectively. Finally, section five presents the main conclusions and directions towards future developments.

\section{A MODEL FOR MULTI-LEGGED LOCOMOTION}

We consider a longitudinal walking system with $2 n$ legs $(n \geq 2)$, with the legs equally distributed along both sides of the robot body, having each one two rotational joints.

Motion is described by means of a world coordinate system (Fig. 1). Defining the $i^{\text {th }}$ leg lengths $L_{i 1}$ and $L_{i 2}$, the body velocity $V_{F}$, the duty factor $\beta$, the transference time $t_{T}=(1-\beta) T$, the support time $t_{S}=\beta T$, the step length $L_{S}$, the stroke pitch $S_{P}$, the body height $H_{B}$, maximum foot clearance $F_{C}$ and the foot trajectory offset $O_{i}(i=1, \ldots, 2 n)$ we consider a periodic trajectory for each foot, with cycle time $T=L_{S} / V_{F}$.

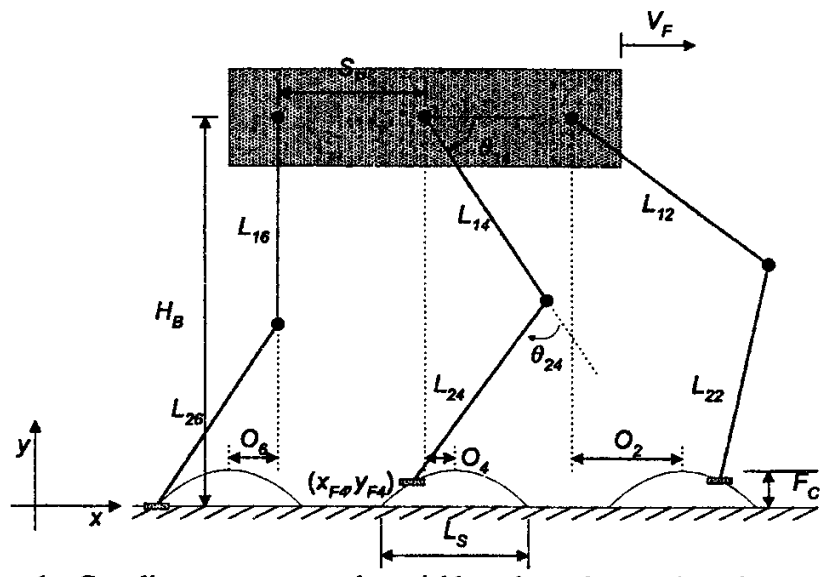

Fig. 1. Coordinate system and variables that characterize the motion trajectories of the multi-legged robot

The algorithm for the forward motion planning accepts the body and $i^{\text {th }}$ feet cartesian trajectories in $\mathbf{p}(t)=\left[x_{i}(t), y_{i}(t)\right]^{\mathrm{T}}$ as inputs and, by means of an inverse kinematics algorithm, generates the related joint trajectories $\theta(t)=\left[\theta_{i 1}(t), \theta_{i 2}(t)\right]^{\mathrm{T}}$, selecting the solution corresponding to a forward knee.

The body of the robot, and by consequence the legs hips, are assumed to have a horizontal movement with a constant forward speed $V_{F}$. Therefore, for leg $i$ the cartesian coordinates of the hip of the legs are given by: 


$$
\begin{gathered}
x_{h i}(t)=V_{F} \cdot t \\
y_{h i}(t)=H_{B}
\end{gathered}
$$

Given a particular gait and duty factor $\beta$, through [13] it is possible to calculate for leg $i$ the corresponding phase $\phi_{i}$ and the time instant where each leg leaves and returns to contact with the ground. From these results, and knowing $T, \beta$ and $t_{S}$, the cartesian trajectories of the tip of the foots must be completed during $t_{T}$.

For each cycle the trajectory of the tip of the swing leg is computed through a cycloid function given by (considering, for example, that the transfer phase starts at $t=0 \mathrm{sec}$ for leg 1), with $f=1 / T$ :

- during the transfer phase:

$$
\begin{gathered}
x_{F 1}(t)=V_{F}\left[t-\frac{1}{2 \pi f} \sin (2 \pi f t)\right] \\
y_{F 1}(t)=\frac{F_{C}}{2}[1-\cos (2 \pi f t)]
\end{gathered}
$$

- during the stance phase:

$$
\begin{gathered}
x_{F 1}(t)=V_{F}\left[T-\frac{1}{2 \pi f} \sin (2 \pi f T)\right]=V_{F} T \\
y_{F 1}(t)=0
\end{gathered}
$$

From the coordinates of the hip and feet of the robot it is possible to obtain the leg joint position and velocities using the inverse kinematics:

$$
\begin{aligned}
\boldsymbol{\theta}(t) & =f[\mathbf{p}(t)] \\
\mathbf{p}(t)=\left[\begin{array}{l}
x_{i}(t) \\
y_{i}(t)
\end{array}\right] & =\left[\begin{array}{l}
x_{h i}(t)-x_{F i}(t) \\
y_{h i}(t)-y_{F i}(t)
\end{array}\right] \\
\dot{\theta}_{i}(t) & =\mathbf{J}^{-1}[\dot{\mathbf{p}}(t)]
\end{aligned}
$$

Based on this data, the trajectory generator is responsible for producing a motion that synchronises and co-ordinates the legs. In order to avoid the impact and friction effects we impose null velocities of the feet in the instants of landing and taking off, assuring also the velocity continuity. These joint trajectories can be accomplished also either with a step or a polynomial versus time acceleration profile. After planning the joint trajectories we calculate the inverse dynamics in order to 'map' the kinematics into power consumption. The robot inverse dynamic model is of the form:

$$
\tau=\mathbf{H}(\boldsymbol{\theta}) \ddot{\boldsymbol{\theta}}+\mathbf{c}(\boldsymbol{\theta}, \dot{\boldsymbol{\theta}})+\mathbf{g}(\boldsymbol{\theta})
$$

where $\tau$ is the $n \times 1$ vector of actuator torques, $\theta$ is the $n \times 1$ vector of joint coordinates, $\mathbf{H}(\theta)$ is the $n \times n$ inertia matrix, $c(\theta, \dot{\theta})$ is the $n \times 1$ vector of centrifugal/Coriolis torques and $\mathbf{g}(\theta)$ is the $n \times 1$ vector of gravitational torques.

\section{MEASURES FOR PERFORMANCE EVALUATION}

In mathematical terms, we provide four global measures of the overall performance of the mechanism in an average sense [14],[15].

\section{A. Locomobility Measure}

The motivation for the development of the locomobility index is to apply the concepts of arm manipulability to multilegged walking. This performance measure can be expressed through the Jacobian matrix. In our case, the global index is obtained by averaging the distance among the center of the ellipsoids and its intersections with the desired trajectories of the foot $\left(L_{F}\right)$, over a complete cycle $T$ [13]:

$$
L_{F}=\frac{1}{T} \int_{b}^{T} E_{F}(t) d t
$$

In this perspective, the most suitable trajectory is the one that maximises $L_{F}$.

\section{B. Mean Absolute Power}

The key measure in this analysis is the mean absolute power per travelling distance. It is computed assuming that power regeneration is not available by actuators doing negative work, that is, by taking the absolute value of the power. At a given joint $j$ (each leg has $m=2$ joints) and leg $i$ (since we are adopting as our case study an hexapod, $n=6$ legs), the mechanical power is the product of the motor torque and angular velocity. The global index is obtained by averaging the mechanical absolute power delivered over a period $T$ and a step $L_{S}$ :

$$
P_{a v}=\frac{1}{L_{S}} \cdot \frac{1}{T} \sum_{i=1}^{n} \sum_{j=1}^{m} \int_{0}^{t}\left|\tau_{i j}(t) \cdot \dot{\theta}_{i j}(t)\right| d t
$$

where $\tau$ is the motor torque and $\dot{\theta}$ is the angular velocity of the joint under consideration. The average of the absolute power consumption $P_{a v}$ should be minimised.

\section{Mean Power Dispersion}

Although minimising power appears to be an important consideration, it may occur instantaneous, very high, power demands. In such cases, the average value can be small while the peaks are physically unrealisable. An alternative index is the standard deviation per meter that evaluates the dispersion around the mean absolute power over a complete cycle $T$ and step length:

$$
\begin{gathered}
P_{i}(t)=\sum_{i=1}^{n} \sum_{j=1}^{m} \tau_{i j}(t) \cdot \dot{\theta}_{i j}(t) \\
D_{a v}=\frac{1}{L_{S}} \cdot \sqrt{\frac{1}{T} \int^{T}\left[P_{i}(t)-P_{a v}\right]^{2} d t}
\end{gathered}
$$


where $P_{i}$ is the total instantaneous mechanical power. In this line of thought, the most suitable trajectory is the one that minimizes $D_{a v}$.

\section{Mean Power Lost}

Another optimisation strategy for an actuated system considers the power lost in the joint actuators per $L_{s}$. From this point of view, the index mean power lost per meter can be defined as:

$$
P_{L}=\frac{1}{L_{S}} \cdot \frac{1}{T} \sum_{i=1}^{n} \sum_{j=1}^{m} \int_{b}^{T}\left[\tau_{i j}(t)\right]^{2} d t
$$

The most suitable trajectory is the one that minimizes $P_{L}$.

\section{SIMULATION RESULTS}

To illustrate the use of the preceding concepts, in this section we develop a set of simulation experiments to estimate the influence of several parameters during periodic gaits and to compare the performance measures. Consequently, the multi-legged locomotion was simulated, in order to examine the role of the walking gait versus $\beta, L_{S}, H_{B}$ and $F_{C}$, with $V_{F}=10 \mathrm{~m} / \mathrm{s}, \quad L_{i 1}=L_{i 2}=1 \mathrm{~m}, \quad O_{i}=0 \mathrm{~m}, \quad M_{1}=M_{2}=1 \mathrm{Kg}$, $M_{\mathrm{b}}=36 \mathrm{Kg}, M_{\mathrm{f}}=0 \mathrm{Kg}$ and $S_{P}=1 \mathrm{~m}$.

Due to the high number of parameters and values, in the next sub-sections we capture the optimal values by crossrelating several distinct combinations.

\section{A. Duty Factor vs Step Length}

Figure 2 depicts the three indices versus $\left(\beta, L_{s}\right)$. We conclude that $P_{a v}, D_{a v}$ and $P_{L}$ increase monotonically with $\beta$ and decrease with $L_{S}$.

\section{B. Body Height vs Step Length}

Figure 3 shows $P_{a v}, D_{a v}$ and $P_{L}$ versus $\left(L_{S}, H_{B}\right)$. We verify that the indices decrease slightly with $H_{B}$ and $L_{S}$.

\section{Duty Factor vs. Foot Clearance}

Figure 4 depicts $P_{a x}\left(\beta, F_{C}\right)$ revealing that it increases with $\beta$ and $F_{C}$. Although not presented $D_{a v}\left(\beta, F_{C}\right)$ and $P_{L}\left(\beta, F_{C}\right)$ show the same type of variation with $\beta$ and $F_{C}$.

The same can be concluded through Figure 5 that depicts $L_{F}\left(\beta, F_{C}\right)$.

In conclusion, comparing all the previous experiments, we can establish a compromise for optimising the Wave Gait namely that the best situation occurs for $\beta \approx 50 \%$, $1.4 \leq H_{B} \leq 1.6 \mathrm{~m}, L_{S}>1.8 \mathrm{~m}$ and $F_{C}<0.1 \mathrm{~m}$, considering $L_{n}=L_{i 2}=1 \mathrm{~m}$ and $O_{i}=0 \mathrm{~m}$.

\section{Body Forward Velocity}

Figure 6 shows $P_{a v}\left(\beta, L_{S}\right)$ for $V_{F}=2 \mathrm{~m} / \mathrm{s}$. Comparing with figure 2, we conclude that $P_{\alpha v}$ increases monotonically with $V_{F}$ without changing the type of the chart. The same occurs with $D_{a v}$ and $P_{L}$.
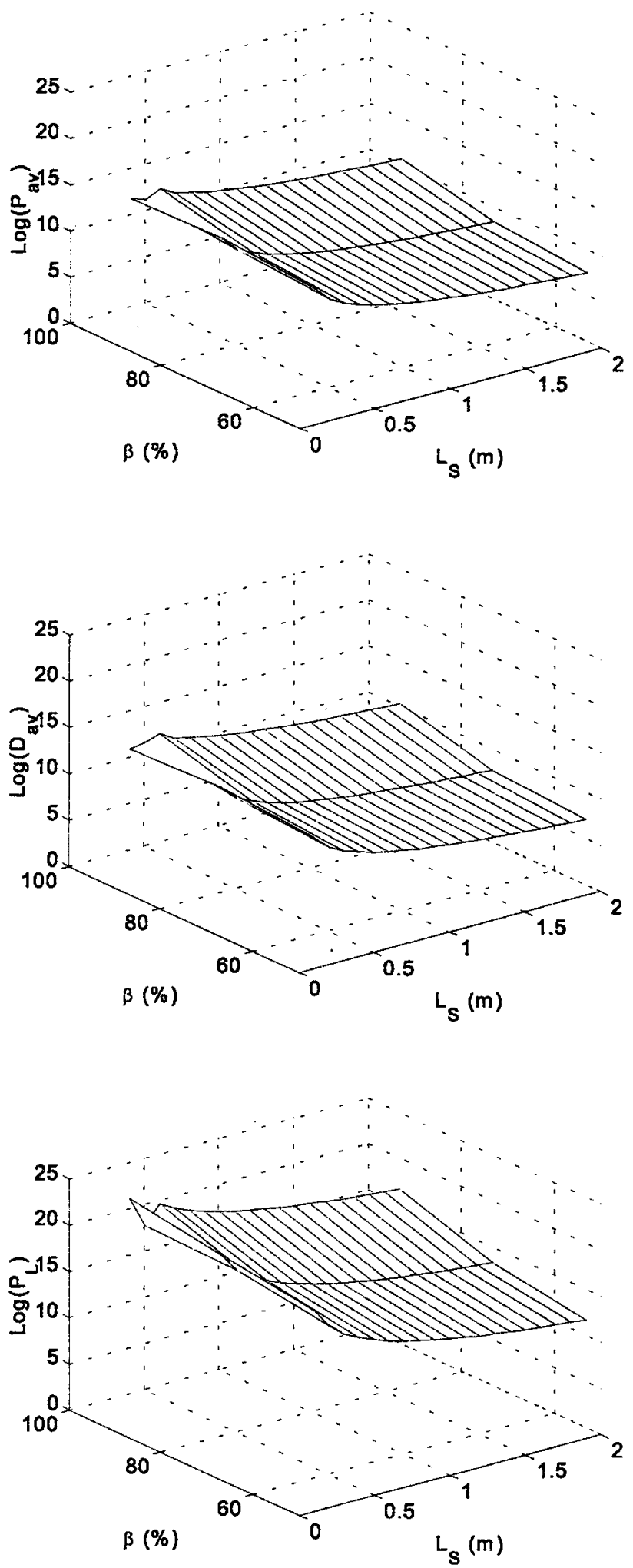

Fig. 2. Plots of $P_{a v}, D_{a v}$ and $P_{L}$ vs. $\left(\beta, L_{S}\right)$ for $F_{C}=0.01 \mathrm{~m}, H_{B}=1.7 \mathrm{~m}$, $V_{F}=10 \mathrm{~m} / \mathrm{s}, W G$. 

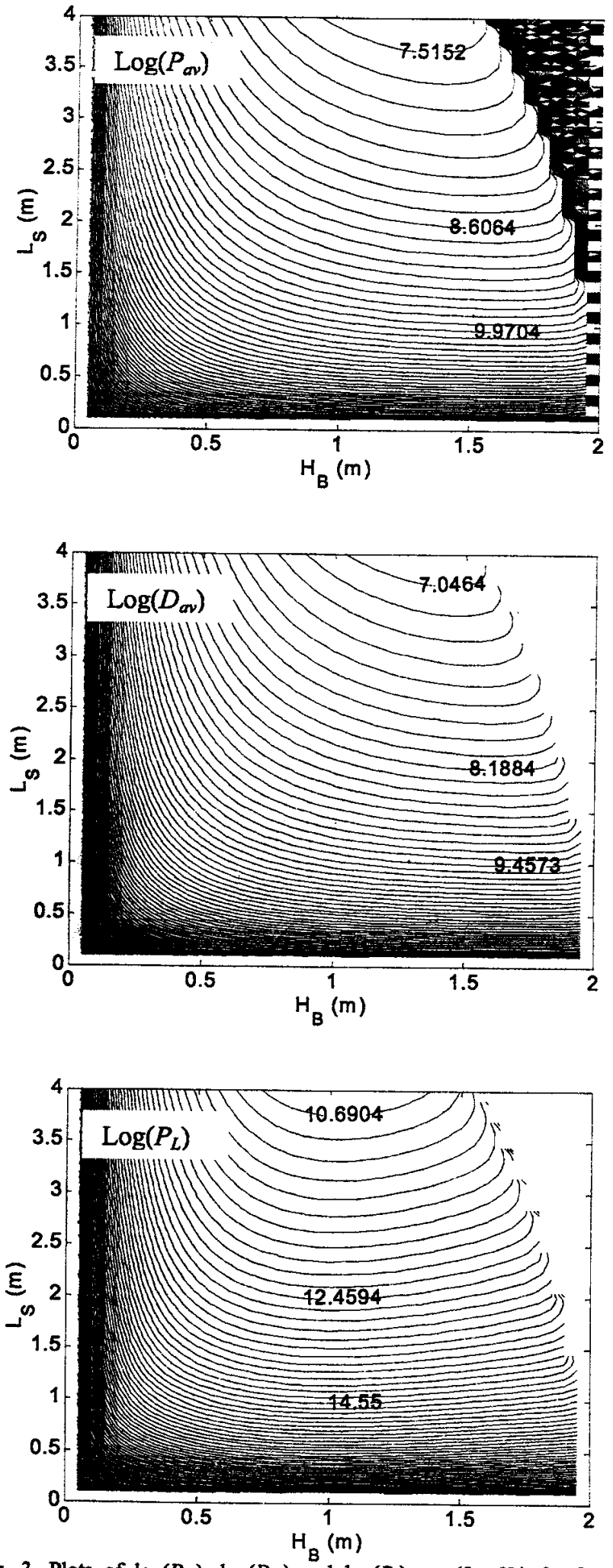

Fig. 3. Plots of $\log \left(P_{a v}\right), \log \left(D_{a v}\right)$ and $\log \left(P_{L}\right)$ vs. $\left(L_{S}, H_{B}\right)$ for $\beta=50 \%$, $F_{C}=0.01 \mathrm{~m}, V_{F}=10 \mathrm{~m} / \mathrm{s}, W G$.

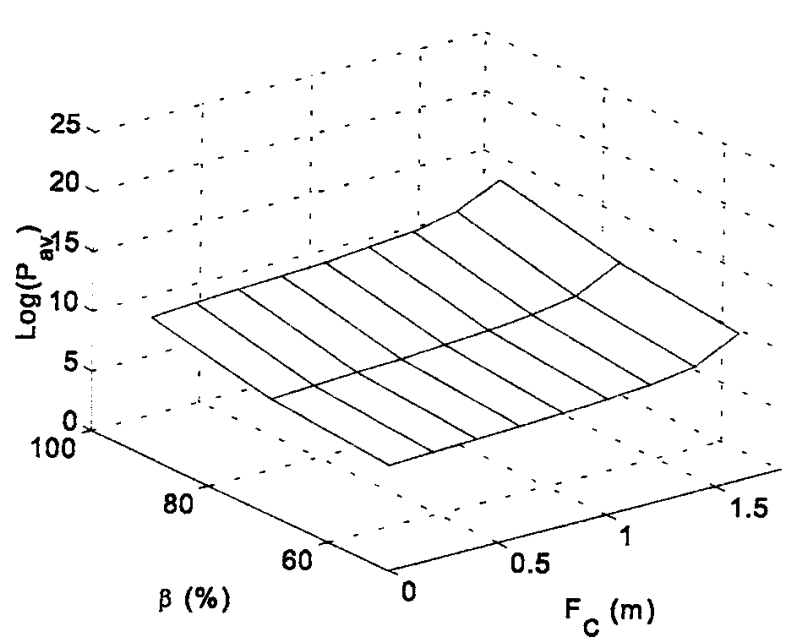

Fig. 4. Plot of $P_{a v}$ vs. $\left(\beta, F_{C}\right)$ for $L_{S}=1.9 \mathrm{~m}, H_{B}=1.7 \mathrm{~m}, V_{F}=10 \mathrm{~m} / \mathrm{s}$, WG.

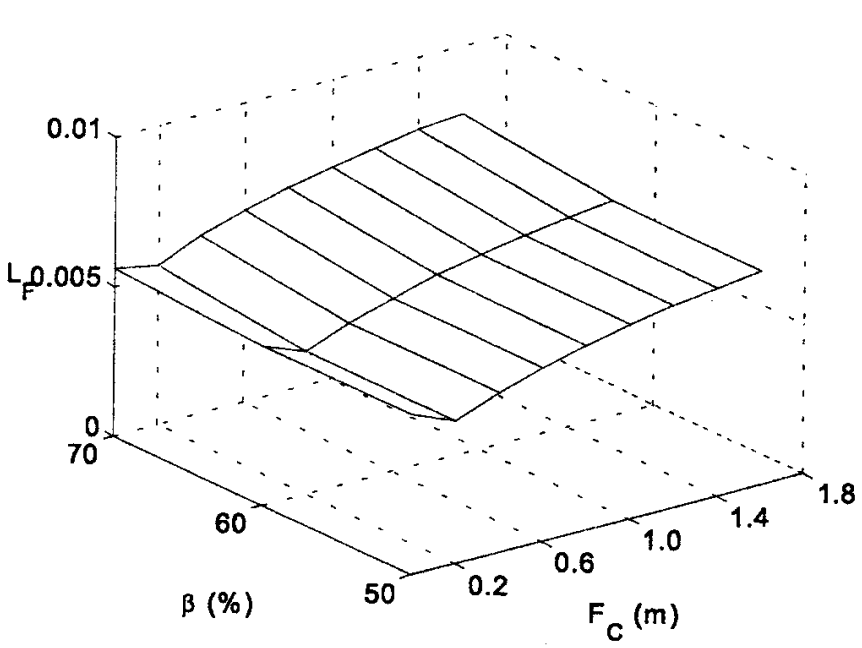

Fig. 5. Plot of $L_{F} v s .\left(\beta, F_{C}\right)$ for $L_{S}=1.9 \mathrm{~m}, H_{B}=1.7 \mathrm{~m}, V_{F}=10 \mathrm{~m} / \mathrm{s}, W G$.

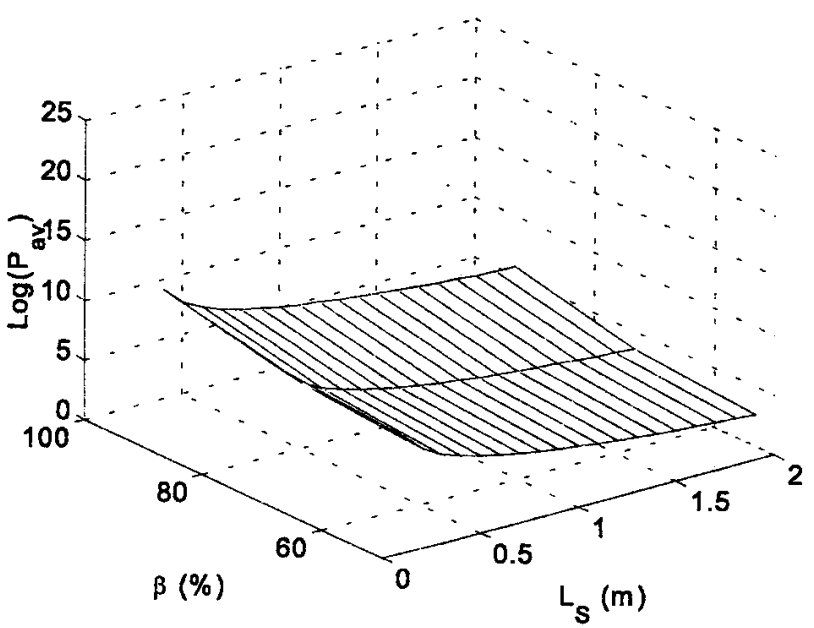

Fig. 6. Plot of $\log \left(P_{a v}\right)$ vs. $\left(\beta, L_{s}\right)$ for $F_{C}=0.01 \mathrm{~m}, H_{B}=1.6 \mathrm{~m}, V_{F}=2 \mathrm{~m} / \mathrm{s}$, WG. 


\section{E. Foot Trajectory Offset vs. Leg Length}

On the previous experiments we considered $L_{i 1}=L_{i 2}=1 \mathrm{~m}$ and $O_{i}=0 \mathrm{~m}$. Now we study the effects of the influence of $L_{i 1}$ (considering $L_{i 1}+L_{i 2}=2 \mathrm{~m}$ ) and $O_{i}$ upon $L_{F}, P_{a v}, D_{\sigma v}$ and $P_{L}$.

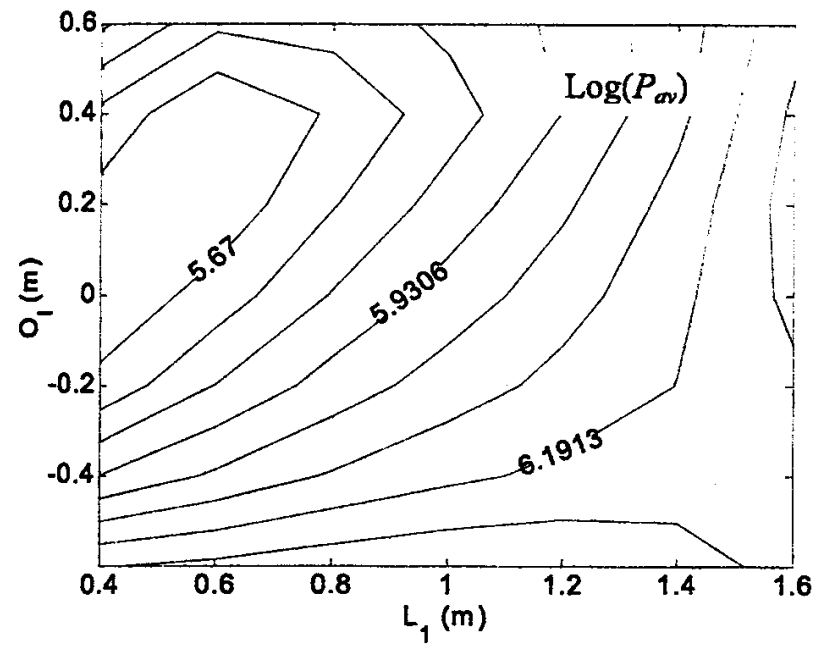

Fig. 7. Plot of $\log \left(P_{\alpha v}\right)$ vs. $\left(O_{i}, L_{i 1}\right)$ for $\beta=50 \%, L_{S}=1.8 \mathrm{~m}, F_{C}=0.01 \mathrm{~m}$, $H_{B}=1.7 \mathrm{~m}, V_{F}=10 \mathrm{~m} / \mathrm{s}, W G$
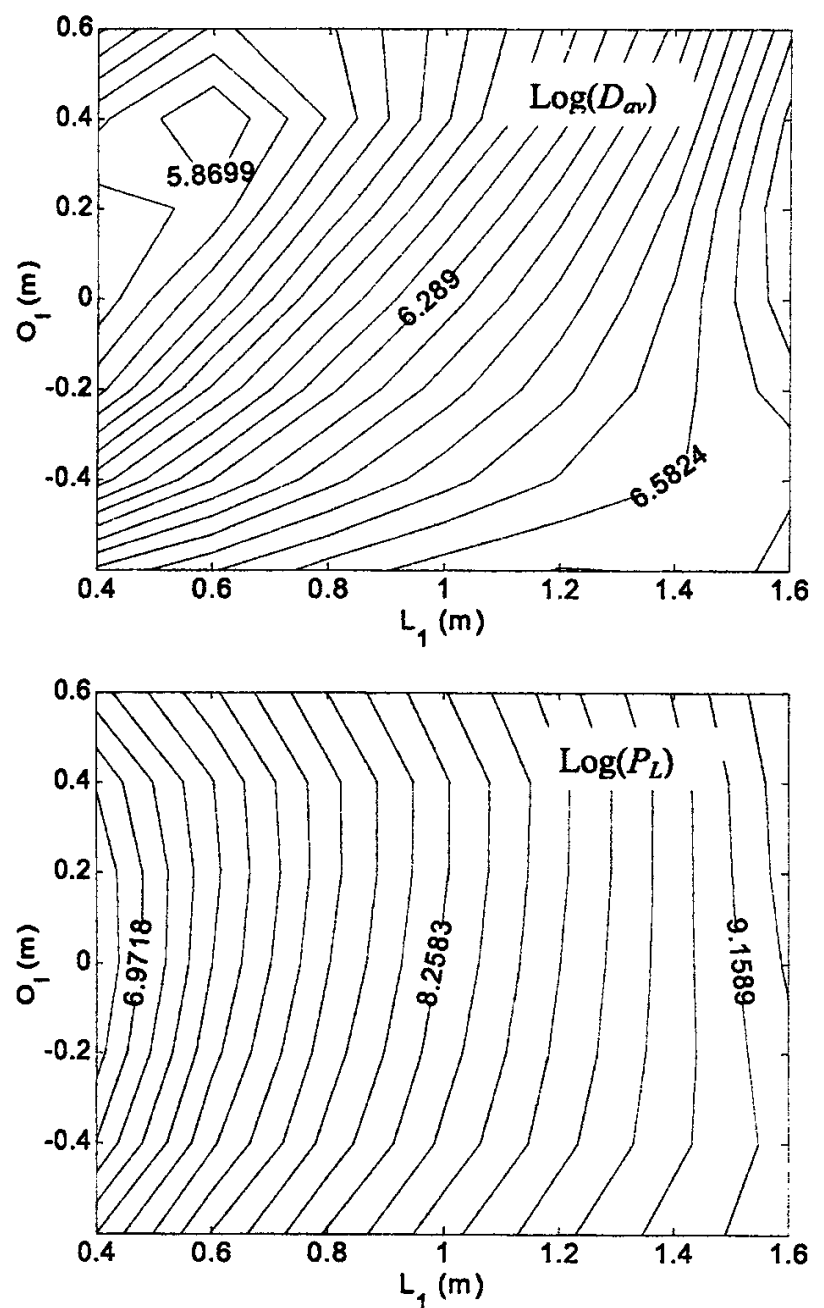

Fig. 8. Plots of $\log \left(D_{w v}\right)$ and $\log \left(P_{L}\right)$ vs. $\left(O_{b}, L_{i 1}\right)$ for $\beta=50 \%, L_{S}=1.8 \mathrm{~m}$, $F_{C}=0.01 \mathrm{~m}, H_{B}=1.7 \mathrm{~m}, V_{F}=10 \mathrm{~m} / \mathrm{s}, W G$.
Figure 7 shows $P_{a v}\left(O_{i,} L_{i 1}\right)$ for for $0.4<L_{i 1}<1.6$ and hipfoot offset of $-0.6<O_{i}<0.6$. We conclude that $P_{\sigma v}$ increases slightly with $L_{i l}$. For values of $O_{i}$ and $L_{i 1}$ outside this interval $P_{\alpha v}$ increases rapidly.

Figure 8 presents $\log \left(D_{a v}\right)$ and $\log \left(P_{L}\right)$. In this case we conclude that the locomotion is more efficient for $L_{i 1} \approx 0.4 \mathrm{~m}$ $\left(L_{i 1}+L_{i 2}=2 \mathrm{~m}\right)$ and $O_{i} \approx 0 \mathrm{~m}$. The same can be concluded through Figure 9 that depicts $L_{F}\left(O_{i}, L_{i 1}\right)$.

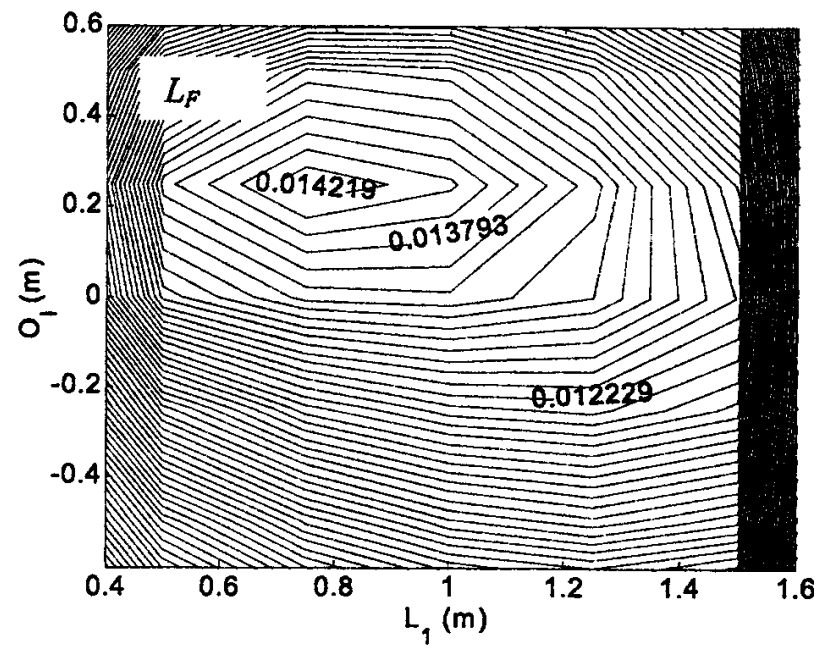

Fig. 9. Plot of $L_{F}$ vs. $\left(O_{i b} L_{i}\right)$ for $\beta=50 \%, L_{S}=1.8 \mathrm{~m}, F_{C}=0.01 \mathrm{~m}, H_{B}=1.7$ $\mathrm{m}, V_{F}=10 \mathrm{~m} / \mathrm{s}, W G$.

\section{CONCLUSIONS}

In this paper we have compared various dynamic aspects of multi-legged robot locomotion gaits. By implementing different motion patterns, we estimated how the robot responds to a variety of locomotion variables such as duty factor, step length, body height, maximum foot clearance, foot trajectory offset and leg lengths. For analysing the system performance four quantitative measures were defined: the foot locomobility index, the average power consumption, the power consumption standard deviation and the power expenditure in the actuators. Through the analysis of the results of the simulations we draw some conclusions on the best set of locomotion variables. Comparing all the experiments we conclude that the results obtained through the indices are compatible.

While our focus has been on a power analysis in periodic gaits, certain aspects of locomotion are not necessarily captured by the proposed measures. Consequently, future work in this area will address the refinement of our models to incorporate more unstructured terrains, namely with distinct trajectory planning concepts. Moreover, we will also address the effects of the foot-ground interaction and a model describing the ground characteristics. The contact and reaction forces at the robot feet will enable further insight towards the development of efficient multi-legged locomotion robots. 


\section{REFERENCES}

[1] Song, S.-M., Waldron, K.J.: Machines that Walk: The Adaptive Suspension Vehicle. The MIT Press, 1989.

[2] Wettergreen, D., Thorpe, C.: Gait Generation for Legged Robots. IEEE Int. Conf. on Intelligent Robots and Systems, 1992.

[3] Jiménez, M. A., Santos, P. G.: Terrain-Adaptive Gait for Walking Machines. The Int. J. of Robotics Research, Vol. 16, n. 3, pp. 320-339, 1997.

[4] Manko, D. J.: A General Model of Legged Locomotion on Natural Terrain. Kluwer, Westinghouse Electric Corporation, 1992.

[5] Venkataraman, S. T.: A Model of Legged Locomotion Gaits. IEEE Int Conf, on Robotics and Automation, USA, 1996

[6] Wettergreen, D., Pangels, H., Bares, J.: Behavior-based Gait Execution for the Dante II Walking Robot. IEEE Int. Conf. on Intelligent Robots and Systems, USA, 1995.

[7] Gregorio, P., Ahmadi, M., Buehler, M.: Design, Control, and Energetics of an Electrically Actuated Legged Robot. IEEE Trans. on Systems, Man and Cybernetics, Vol. 27, n. 4, 1997.

[8] Lapshin, V. V.: Energy Consumption of a Walking Machine. Model Estimations and Optimization. IEEE Int. Conf. on Advanced Robotics, Hungary, pp. 420-425, 1995.
[9] Caux, S., Zapata, R.: Modeling and Control of Biped Robot Dynamics. Robotica, Vol. 17, pp. 413-426, 1997.

[10] Nelson, G. M.: Modeling and Simulation of an Insect-like Hexapod. MSc. Thesis, Dept. of Mechanical and Aerospace Engineering, Case Western Reserve University, 1995.

[11] Nelson, G. M, Quinn, R. D.: Posture Control of a Cockroach-like Robot. IEEE Control Systems, Volume 19, Number 2, pp. 9-14, 1999.

[12] Pfeiffer, F., Elze, J., Weidemann, H.-J.: The TUM Walking Machine. Intelligent Automation and Soft Computing, Vol.1, No. 3, pp. 307-323, 1995.

[13] Silva, M. F., Machado, J. A. T., Lopes, A. M.: Performance Analysis of Periodic Gaits in Multi-Legged Locomotion. IEEE Int. Conf. on Advanced Robotics, Hungary, 2001.

[14] Silva, M. F., Machado, J. A. T., Lopes, A. M.: Energy Analysis of Multi-Legged Locomotion Systems. $4^{\text {th }}$ Int. Conf. on Climbing and Walking Robots, Germany, 2001.

[15] Silva F, M. Machado, J. A. T.: Energy Analysis During Biped Walking. IEEE Int. Conf. on Robotics and Automation, USA, 1999.

[16] Silva, F. M., Machado, J. A. T.: Towards Efficient Biped Robots. IEEE Int. Conf. on Inteliligent Robots and Systems, Canada, 1998. 